\title{
The endocannabinoid anandamide has an anti-inflammatory effect on CCL2 expression in vascular smooth muscle cells
}

\author{
Beatrice Pflüger-Müller ${ }^{1,2}$. James A. Oo ${ }^{1,2}$. Jan Heering ${ }^{4}$ Timothy Warwick ${ }^{1,2}$. Ewgenij Proschak ${ }^{6}$. \\ Stefan Günther ${ }^{3}$. Mario Looso ${ }^{3}$. Flávia Rezende ${ }^{1,2}$. Christian Fork ${ }^{1,2}$. Gerd Geisslinger ${ }^{4,5}$. Dominique Thomas ${ }^{5}$. \\ Robert Gurke $^{4,5} \cdot$ Dieter Steinhilber $^{4,6} \cdot$ Marcel Schulz $^{7} \cdot$ Matthias S. Leisegang $^{1,2} \cdot$ Ralf P. Brandes $^{1,2}$
}

Received: 17 January 2020 / Accepted: 14 April 2020 / Published online: 22 April 2020

(c) The Author(s) 2020

\begin{abstract}
Endocannabinoids are important lipid-signaling mediators. Both protective and deleterious effects of endocannabinoids in the cardiovascular system have been reported but the mechanistic basis for these contradicting observations is unclear. We set out to identify anti-inflammatory mechanisms of endocannabinoids in the murine aorta and in human vascular smooth muscle cells (hVSMC). In response to combined stimulation with cytokines, IL-1 $\beta$ and TNF $\alpha$, the murine aorta released several endocannabinoids, with anandamide (AEA) levels being the most significantly increased. AEA pretreatment had profound effects on cytokine-induced gene expression in hVSMC and murine aorta. As revealed by RNA-Seq analysis, the induction of a subset of 21 inflammatory target genes, including the important cytokine CCL 2 was blocked by AEA. This effect was not mediated through AEA-dependent interference of the AP-1 or NF- $\mathrm{kB}$ pathways but rather through an epigenetic mechanism. In the presence of AEA, ATAC-Seq analysis and chromatin-immunoprecipitations revealed that CCL2 induction was blocked due to increased levels of $\mathrm{H} 3 \mathrm{~K} 27 \mathrm{me} 3$ and a decrease of $\mathrm{H} 3 \mathrm{~K} 27 \mathrm{ac}$ leading to compacted chromatin structure in the CCL2 promoter. These effects were mediated by recruitment of HDAC4 and the nuclear corepressor NCoR1 to the CCL2 promoter. This study therefore establishes a novel anti-inflammatory mechanism for the endogenous endocannabinoid AEA in vascular smooth muscle cells. Furthermore, this work provides a link between endogenous endocannabinoid signaling and epigenetic regulation.
\end{abstract}

Keywords Anandamide $\cdot$ HDAC4 $\cdot$ NCoR1 $\cdot$ Inflammation $\cdot$ CCL2

Electronic supplementary material The online version of this article (https://doi.org/10.1007/s00395-020-0793-3) contains supplementary material, which is available to authorized users.

Ralf P. Brandes

brandes@vrc.uni-frankfurt.de

1 Fachbereich Medizin, Institute for Cardiovascular Physiology, Goethe University, Theodor-Stern-Kai 7, 60590 Frankfurt, Germany

2 German Center for Cardiovascular Research (DZHK), Partner site Rhein Main, Frankfurt, Germany

3 Max-Planck-Institute for Heart- and Lung Research (MPI-HLR), 61231 Bad Nauheim, Germany

4 Branch for Translational Medicine and Pharmacology TMP, Fraunhofer Institute for Molecular Biology and Applied Ecology IME, 60438 Frankfurt, Germany

\section{Introduction}

Endocannabinoids are unsaturated fatty acid derivatives that represent an important class of signaling lipid mediators best known for their effects in the central nervous system

5 Faculty of Medicine, Pharmazentrum Frankfurt/ZAFES, Institute of Clinical Pharmacology, Goethe-University, 60590 Frankfurt, Germany

6 Institute of Pharmaceutical Chemistry, Goethe-University, 60438 Frankfurt, Germany

7 Vascular Research Centre, Goethe-University, 60596 Frankfurt, Germany 
[18]. It is well appreciated that endocannabinoids also have numerous effects directly on the cardiovascular system [26, 27]: endocannabinoids mediate intercellular and bidirectional communication in the brain [23], alter the vasomotor response [34] and cellular gene expression. The effects of endocannabinoids are largely mediated by the $\mathrm{G}_{\mathrm{i}}$-coupled receptors $\mathrm{CB} 1$ and $\mathrm{CB} 2[6,22]$ and to some extent by nuclear receptors like PPARs, which have been shown to be activated by endocannabinoids [25]. As endocannabinoids are released and taken up by different cells, these receptors can mediate autocrine and paracrine effects [10]. Since many cells, including those of the vascular system, produce and degrade endocannabinoids, these compounds have become interesting targets for the treatment and prevention of cardiovascular disease. Numerous studies have looked into the vascular consequences of a genetic knockout or activation of different endocannabinoid receptors and producing and degrading enzymes, with focus on inflammatory responses and atherosclerosis [24, 30]. However, the results were complex in that no unifying picture emerged. This is, in part, a consequence of the fact that the receptors all have different functions and that usually more than one endocannabinoid is produced by a cell. Depending on the presence of degrading and producing enzymes, cells can simultaneously produce 2-arachidonylglycerol (2-AG), arachidonoyl-ethanolamine (AEA), also known as anandamide, palmitoylethanolamide (PEA) and oleoylethanolamine (OEA) and each of these endocannabinoids can activate multiple receptors and signaling cascades.

In the present study we set out to identify novel signaling mechanisms that are activated by endocannabinoids in vascular smooth muscle cells. We found that AEA has a unique anti-inflammatory function which is mediated by an interesting epigenetic mechanism.

\section{Materials and methods}

\section{Reagents}

The following reagents were used: Human recombinant Interleukin-1 (IL-1)- $\beta$ (\#200-01B, Peprotech), murine recombinant Interleukin-1 (IL-1)- $\beta$ (\#211-11B, Peprotech), human recombinant tumor necrosis factor (TNF)- $\alpha$ (\#300$01 \mathrm{~A}$, Peprotech), murine recombinant tumor necrosis factor (TNF)- $\alpha$ (\#315-01A, Peprotech) Lipopolysaccharide from E-coli (\#L3024, Sigma), Collagen Type I Rat Tail (\#354236, Corning Incorporated), $N$-arachidonoylethanolamine (Anandamide, AEA, Sigma-Aldrich, \#A-0580-25MG), RGFP966 (\#16917.00, Cayman Chemicals), MC1568 (27761-1, BPS Bioscience), Diclofenac sodium salt (\#D6899, Sigma).

The used antibodies can be find in supplemental Table 4 . For Western blot antibodies were diluted 1:1000. For immunofluorescence and PLA antibodies were diluted in standard way 1:500 except for HDAC4 1:1000. ChIP experiments were performed with standard $3 \mu \mathrm{g}$ of antibody except for NCoR1 were we used $6 \mu$. Primers for RT-qPCR, used siRNA and primers for ChIP PCRs are listed in Supplemental Tables 1, 2 and 3.

\section{Cell stimulation}

HAoSMC and freshly isolated murine aortic smooth muscle cells were exposed to media with L-glutamine and $1 \%$ FCS overnight or for at least $16 \mathrm{~h}$. The next day cells were incubated in media with $1 \%$ FCS, L-glutamine and $10 \mu \mathrm{M}$ diclofenac (Sigma Aldrich, \#D-6899) for $1 \mathrm{~h}$ and treated with $100 \mathrm{nM}$ AEA or EtOH as solvent for $2.5 \mathrm{~h}$. Diclofenac was used to prevent cyclooxygenase-mediated breakdown of AEA. Subsequently, $10 \mathrm{ng} / \mathrm{mL}$ IL- $1 \beta$, TNF $\alpha(10 \mathrm{ng} / \mathrm{mL}$ or LPS $(10 \mu \mathrm{g} / \mathrm{mL})$ was added for $1.5 \mathrm{~h}$. For protein measurements $100 \mathrm{nM}$ AEA $(1 \mathrm{~h})$ and $10 \mathrm{ng} / \mathrm{mL}$ IL- $1 \beta$ treatment $(3 \mathrm{~h})$ in the presence of $10 \mu \mathrm{M}$ diclofenac was used. For immunofluorescence and PLA measurements $100 \mathrm{nM}$ AEA $2.5 \mathrm{~h}$ and $1 \mathrm{~h} 10 \mathrm{ng} / \mathrm{mL}$ IL- $1 \beta$ treatment in the presence of $10 \mu \mathrm{M}$ diclofenac was used. Murine aortic rings were cultured in media without supplements and with $0.1 \%$ BSA. Treatment was performed in the presence of $10 \mu \mathrm{M}$ diclofenac and pre-treatment with $100 \mathrm{nM}$ AEA overnight. The next day, murine IL- $1 \beta(10 \mathrm{ng} / \mathrm{mL})$, murine $\mathrm{TNF} \alpha$ $(10 \mathrm{ng} / \mathrm{mL})$ or LPS $(10 \mu \mathrm{g} / \mathrm{mL})$ was added and incubated for $1.5 \mathrm{~h}$.

\section{Cell culture}

Human aortic smooth muscle cells (HAoSMC) (\#35405a) were purchased from PELOBiotech (Planegg, Germany). The cells were cultured on collagen-coated dishes in PELOBiotech Smooth Muscle Cell Medium (\#PB-MH200-2190) with $8 \%$ heat-inactivated fetal calf serum (FCS), L-glutamine, penicillin $(50 \mathrm{U} / \mathrm{ml})$ and streptomycin $(50 \mu \mathrm{g} /$ ml) (\#15140-122, Gibco lifetechnologies, Carlsbad, CA, USA), EGF, bFGF and insulin from singlequots (PELOBiotech, Planegg, Germany) in a humidified atmosphere of 5\% $\mathrm{CO}_{2}$ at $37^{\circ} \mathrm{C}$. For experiments cells from passage 5 to 10 were used. For each experiment at least three to five different batches (each a different donor) of HAoSMC were used. After siRNA transfection cells were transferred to media without penicillin/streptomycin.

\section{Experimental animals}

All experimental procedures were approved by the local governmental authorities and were performed in accordance with the animal protection guidelines. Mice were housed in a specified pathogen-free facility with $12 \mathrm{~h}$ light-dark 
cycle and free access to chow and water. For these studies 4-6 weeks old male mice of the C57BL/6J strain were used.

\section{Cell isolation of murine aortic smooth muscle cells and organ culture}

Mice (four animals per preparation) were killed with isoflurane overdose, aortas were removed and adhering connective tissue removed. For organ culture, the aorta was cut into $1 \mathrm{~mm}$ rings. These were cultured for $16 \mathrm{~h}$ in endothelial basal medium (EBM) with $0.1 \% \mathrm{BSA}, 10 \mu \mathrm{M}$ diclofenac and treated as already described. For cell isolation, vessels were cut open, the endothelium was scraped of and the tissue was extensively washed. Subsequently, the aortae were minced in $50 \mu \mathrm{L}$ sterile MCDB medium with $0.1 \%$ BSA into $1 \mathrm{~mm}$ small pieces and subjected to collagenase dissociation $(380 \mathrm{U} / \mathrm{mL})$ for $40-60 \mathrm{~min}$ at $37{ }^{\circ} \mathrm{C}$ with trituration every $10 \mathrm{~min}$. The supernatant of this procedure was pelleted and cultured in SMC basal medium from PELOBiotech (\#PB-MH-200-2190) with 8\% FCS and insulin in fibronectin coated dishes.

\section{Monocyte migration: Boyden chamber}

THP-1 cells were cultured in RPMI medium 1640 + GlutaMAX from Gibco supplemented with $8 \%$ fetal calf serum (FCS), $10 \mathrm{mM}$ HEPES, $1 \mathrm{mM}$ sodium pyruvate (sigma) and streptomycin $(50 \mu \mathrm{g} / \mathrm{mL})$ in a humidified atmosphere of $5 \% \mathrm{CO}_{2}$ at $37{ }^{\circ} \mathrm{C}$. THP-1 cell migration was studied in a modified Boyden chamber assay using a transwell chamber system (Fluoroblock, $8 \mu \mathrm{M}$ pore size, BD Bioscience, Heidelberg, Germany). $2.5 \times 10^{4}$ cells were seeded in the insert in RPMI medium with $0.1 \%$ BSA. Migration towards the stimulus or supernatant derived from HAoSMC conditioned media which was added to the lower chamber, was determined after $14 \mathrm{~h}$. For conditioned media HAoSMC were treated with $100 \mathrm{nM}$ AEA ( $2 \mathrm{~h})$ and $10 \mathrm{ng} / \mathrm{mL}$ IL- $1 \beta$ $(3 \mathrm{~h})$. For further analysis supernatant was incubated with $50 \mathrm{ng} / \mathrm{mL}$ recombinant human CCL2 (\#300-04, Peprotech) or 1:1000 diluted CCL2 antibody (\#sc-52877, Santa Cruz).

\section{Chromatin immuno-precipitation (ChIP) in HAoSMC}

HAoSMC were incubated in media without supplements and additional $1 \%$ FCS overnight. The next day $10 \mu \mathrm{M}$ diclofenac was added and incubated for $1 \mathrm{~h}$. Afterwards cells were treated with $100 \mathrm{nM}$ AEA for $2 \mathrm{~h}$ and $1 \mathrm{~h}$ with $10 \mathrm{ng} / \mathrm{mL}$ IL-1 $\beta$. Cells were crosslinked with formaldehyde $(1 \%, 10 \mathrm{~min})$. To stop crosslinking glycine $(0.1 \mathrm{M}, 5 \mathrm{~min})$ was added. The cells were washed twice with cold DPBS (without calcium and magnesium), scraped and transferred to Falcon tubes and centrifuged for $5 \mathrm{~min}$ at $800 \times \mathrm{g}$ RT. Nuclear isolation was performed with truCHIP ${ }^{\mathrm{TM}}$ Chromatin
Shearing Kit (Covaris, Woburn, USA) according to the manufacturers protocol. Afterwards, the lysates were sonified with a Bioruptor Plus (ten cycles, 30 s on, 90 s off; Diagenode, Seraing, Belgium) at $4{ }^{\circ} \mathrm{C}$. Debris were removed by centrifugation. Chromatin content was measured by QuBit to adjust the chromatin concentration for each tube. The lysates were diluted $1: 3$ in dilution buffer $(20 \mathrm{mmol} / \mathrm{L}$ Tris/ $\mathrm{HCl} \mathrm{pH}$ 7.4, $100 \mathrm{mmol} / \mathrm{L} \mathrm{NaCl}, 2 \mathrm{mmol} / \mathrm{L}$ EDTA, $0.5 \%$ Triton X-100 and protease inhibitors). After pre-clearing with $25 \mu \mathrm{L}$ DiaMag protein A-coated magnetic beads (Diagenode, Seraing, Belgium) for $1 \mathrm{~h}$ at $4{ }^{\circ} \mathrm{C}$, samples were incubated overnight at $4{ }^{\circ} \mathrm{C}$ with $3 \mu \mathrm{g}$ of antibody. $5 \%$ of the samples served as input. The antibody complexes were collected with $50 \mu \mathrm{L}$ DiaMag protein A-coated magnetic beads (Diagenode, Seraing, Belgium) for $3 \mathrm{~h}$ at $4{ }^{\circ} \mathrm{C}$, subsequently washed twice for 10 min with each of the wash buffers 1-3 (Wash Buffer 1: $20 \mathrm{mmol} / \mathrm{L}$ Tris/HCl pH 7.4, $150 \mathrm{mmol} / \mathrm{L} \mathrm{NaCl}, 0.1 \%$ SDS, $2 \mathrm{mmol} / \mathrm{L}$ EDTA, $1 \%$ Triton X-100; Wash Buffer 2: $20 \mathrm{mmol} / \mathrm{L} \mathrm{Tris} / \mathrm{HCl} \mathrm{pH} \mathrm{7.4,} 500 \mathrm{mmol} / \mathrm{L} \mathrm{NaCl}, 2 \mathrm{mmol} / \mathrm{L}$ EDTA, 1\% Triton X-100; Wash Buffer 3: $10 \mathrm{mmol} / \mathrm{L}$ Tris/ $\mathrm{HCl} \mathrm{pH} 7.4,250 \mathrm{mmol} / \mathrm{L}$ lithium chloride, $1 \%$ Nonidet, $1 \%$ sodium deoxycholate, $1 \mathrm{mmol} / \mathrm{L}$ EDTA) and finally washed with TE-buffer $\mathrm{pH}$ 8.0. Elution of the beads was performed with elution buffer $(0.1 \mathrm{M} \mathrm{NaHCO} 3,1 \%$ SDS $)$ containing $1 \times$ proteinase $\mathrm{K}$ (Diagenode, Seraing, Belgium) and shaking at $600 \mathrm{rpm}$ for $1 \mathrm{~h}$ at $55^{\circ} \mathrm{C}, 1 \mathrm{~h}$ at $62{ }^{\circ} \mathrm{C}$ and $10 \mathrm{~min}$ at $95{ }^{\circ} \mathrm{C}$. Subsequently, the eluate was purified with the QiaQuick PCR purification kit (Qiagen, Hilden, Germany) and subjected to qPCR analysis (qPCR Primers: Supplemental Table 3, antibodies: Supplemental Table 4).

\section{Immunofluorescence staining in HAoSMC}

HAoSMC were treated as already described for ChIP experiments. Cells were fixed by adding paraformaldehyde in PBS to a final concentration of $2 \%$ for $10 \mathrm{~min}$. Cells were permeabilized with $0.05 \%$ Triton X-100 for 10 min and blocked for 30 min with 3\% BSA. The NCoR1 antibody (\#A301145A) or HDAC4 (\#A303-467A) was incubated overnight at $4{ }^{\circ} \mathrm{C}$. The secondary antibody (incubation $1 \mathrm{~h}$ ) and DAPI staining (incubation $10 \mathrm{~min}$ ) were performed the next day after several washing steps with PBS and 0.3\% Tween. Cells were stored in PBS with $0.02 \%$ sodium azide. Images were acquired with a Zeiss LSM800 laser scanning microscope.

\section{Proximity ligation assay}

Human aortic smooth muscle cells were seeded onto 8-well ibidi slides. The next day, cells were treated for $2.5 \mathrm{~h}$ with $100 \mathrm{nM}$ AEA and subsequently for $1 \mathrm{~h}$ with $10 \mathrm{ng} / \mathrm{mL}$ IL-1 $\beta$. All experiments were done with preincubation of $10 \mu \mathrm{M}$ diclofenac. Cells were fixed and permeabilized as already described for immunofluorescence and blocked with 
blocking buffer of the Duolink In Situ Detection Reagents Orange Kit (\#DU92007, Sigma Aldrich). Incubation with primary antibodies against HDAC4 (rabbit, A303-467A, Bethyl) and NCoR1 (LSBio, \#LS-C108878-100) was performed overnight at $4{ }^{\circ} \mathrm{C}$. The next day after washing with PBS and $0.3 \%$ Tween, samples were incubated with the PLA-probes (Duolink In Situ PLA Probes anti-mouse plus, \#DUO92001 and anti-rabbit minus, \#DUO92005, SigmaAldrich) for $1 \mathrm{~h}$ at $37^{\circ} \mathrm{C}$, washed and ligated for $30 \mathrm{~min}$ at $37^{\circ} \mathrm{C}$. After additional washing steps the amplification with polymerase took place for $100 \mathrm{~min}$ at $37{ }^{\circ} \mathrm{C}$. After staining the nuclei with DAPI the cells were washed several times and stored in PBS with $0.02 \%$ sodium azide. Images were acquired with a Zeiss LSM800 laser scanning microscope.

\section{siRNA transfection}

Human aortic smooth muscle cells (HAoSMC) were seeded for $50 \%$ confluence and transfected the next day with Lipofectamine 3000 in $8 \%$ FCS (without antibiotics) using $40 \mathrm{nM}$ siRNA for $72 \mathrm{~h}$, with a medium change after $24 \mathrm{~h}$. Scrambled Stealth RNAi ${ }^{\mathrm{TM}}$ (siScr1-3) was used as negative control (Supplemental Table 1). $4 \mathrm{~h}$ prior to experiments, the medium was changed to SMC basal medium from PELOBiotech (\#PB-MH-200-2190) without supplements but with $1 \%$ FCS.

\section{Protein isolation and western blot analysis}

Cells were washed twice with Hank's buffer and lysed with Triton X-100 lysis buffer (20 mM TRIS/Cl pH 7.5, $150 \mathrm{mM}$ $\mathrm{NaCl}, 10 \mathrm{mM}$ NaPi, $20 \mathrm{mM} \mathrm{NaF}, 1 \%$ Triton X-100, $2 \mathrm{mM}$ orthovanadate (OV), $10 \mathrm{nM}$ okadaic acid, protein-inhibitor $\operatorname{mix}$ (PIM), $40 \mu \mathrm{g} / \mathrm{ml}$ phenylmethylsulfonyl fluoride). Cells were centrifuged for $10 \mathrm{~min}$ at full speed at $4^{\circ} \mathrm{C}$. Supernatant was used and after determination of protein concentration by the Bradford assay, equal amounts of protein were boiled in sample buffer and separated by SDS-PAGE gel electrophoresis. The gels were blotted onto a nitrocellulose membrane and blocked in Rotiblock (Carl Roth, Germany). Infrared-fluorescent-dye-conjugated secondary antibodies were purchased from Licor (Bad Homburg, Germany) and detected with an infrared-based laser scanning detection system (Odyssey Classic, Licor, Bad Homburg, Germany). Used antibodies listed in Supplemental Table 4.

\section{Nuclear extraction}

Cells were washed twice with Hank's buffer and detached by scraping. After centrifugation, the pellet was resuspended in buffer A $(10 \mathrm{mM}$ Hepes pH 7.9, $10 \mathrm{mM} \mathrm{KCl}, 0.1 \mathrm{mM}$ EDTA, $0.1 \mathrm{mM}$ EGTA) and incubated for $15 \mathrm{~min}$ on ice. Subsequently, $0.75 \%$ Nonidet (NP-40) was added and the samples centrifuged at full speed for $1 \mathrm{~min}$ at $4{ }^{\circ} \mathrm{C}$. The supernatant was removed as cytosolic fraction. The nuclear fraction was resuspended in buffer $\mathrm{C}(20 \mathrm{mM}$ Hepes $\mathrm{pH}$ 7.9, 0.4 M NaCl, $1 \mathrm{mM}$ EDTA, $1 \mathrm{mM}$ EGTA) additionally with 6.25 units/mL Benzonase and incubated for $15 \mathrm{~min}$ at $25^{\circ} \mathrm{C}$ while shaking. Afterwards the lysates were incubated for $1 \mathrm{~h}$ at $4{ }^{\circ} \mathrm{C}$. After centrifugation 5 min full speed at $4{ }^{\circ} \mathrm{C}$ the nuclear fraction and the pellet (chromatin fraction) were isolated. Used antibodies listed in Supplemental Table 4.

\section{Electrophoretic mobility shift assay (EMSA)}

Human aortic smooth muscle cells were stimulated with $100 \mathrm{nmol} / \mathrm{L}$ AEA, $150 \mathrm{~min}$ and subsequently treated with $10 \mathrm{ng} / \mathrm{mL} \mathrm{IL}-1 \beta, 30 \mathrm{~min}$. Afterwards protein was isolated by nuclear extraction as described before. $5 \mu \mathrm{g}$ nuclear protein was incubated for $30 \mathrm{~min}$ with IRDye pre-labeled oligonucleotides specific for NF-kB consensus sequence (\#82907924, LI-COR). Therefore supplements and instructions of the EMSA kit (\#829-07910, LI-COR) were used. Samples were loaded on 5\% native acrylamide gels and separated for $30 \mathrm{~min}$.

\section{ELISA}

Cells were treated as described before for protein isolation. The supernatant was collected after $4 \mathrm{~h}$. Afterwards the ELISA was performed as written in the instructions of CCL2 ELISA Kit (\#BMS281, Thermofisher).

\section{Quantitative RT-qPCR}

Total RNA was extracted with an RNA Mini Kit (Bio\&SELL). RNA from murine and human aortic tissue was extracted with a specific lysis buffer (4 M Guanidinium thiocyanate, $25 \mathrm{mM}$ Na-citrat), $0.5 \% \mathrm{~N}$-lauryl sarcosine and $\beta$-mercaptoethanol). The tissue was homogenized with a TissueLyser LT (Qiagen) three times for $1 \mathrm{~min}$ each and then centrifuged for $1 \mathrm{~min}$ at full speed. Subsequently the supernatant was further processed with the RNA Mini Kit (Bio\&SELL). cDNA was prepared with SuperScript III reverse transcriptase (Thermo Fisher) and random hexamer (Promega, \#C118A) together with oligo (dT) primers (Sigma-Aldrich, \#O4387). Quantitative real-time PCR was performed with SYBR Green Master Mix and ROX as reference dye (Bio\&SELL \#76.580.5000) in AriaMX Cycler (Agilent). Relative expression of target genes was normalized to GAPDH (or $\alpha$-actin for tissue samples) and analyzed by the delta-delta $\mathrm{Ct}$ method with the AriaMX qPCR software (Agilent Technologies, Santa Clara, CA, USA) (qRTPCR primers: Supplemental Table 2). 


\section{LC-MS/MS}

Endocannabinoids and endocannabinoid-like substances were analyzed by liquid chromatography-electrospray ionization-tandem mass spectrometry (LC-ESI-MS/MS). Minor modifications were made due to the special requirements of tissue samples. In brief, tissue samples were homogenized in water/ethanol 3:1 (v/v) using a Qiagen tissue lyser, resulting in tissue homogenates of $0.04 \mathrm{mg} / \mu \mathrm{L}$. The analytes were extracted by liquid-liquid-extraction with ethyl acetate/hexane 9:1 (v/v) using $200 \mu \mathrm{L}$ tissue homogenate ( $8 \mathrm{mg}$ tissue) after spiking with the respective isotopically labeled internal standards. Chromatographic separation of the analytes was done with an Agilent 1290 Infinity I UHPLC system equipped with an Acquity UPLC BEH C18 column $(100 \times 2.1 \mathrm{~mm}, 1.7 \mu \mathrm{m}$, Waters, Eschborn, Germany). The quantification of all analytes was carried out in a hybrid triple quadrupole-ion trap mass spectrometer QTRAP $6500+$ (Sciex, Darmstadt, Germany) equipped with a Turbo$\mathrm{V}$-source operating in positive ESI mode. For all analytes, the concentrations of the calibration standards, quality controls and samples were evaluated by Analyst software 1.6.3 and MultiQuant software 3.0.2 (Sciex) using the internal standard method (isotope-dilution mass spectrometry). Calibration curves were calculated by linear regression with $1 / x$ weighting.

\section{RNA sequencing}

Total RNA was isolated with the RNA Mini Kit from Bio\&SELL and on-column DNase digestion (DNase-Free DNase Set, Promega) was performed to remove any potential DNA contamination. Total RNA and library integrity were verified with LabChip Gx Touch 24 (Perkin Elmer). $1 \mu \mathrm{g}$ of total RNA was used as input for SMARTer Stranded Total RNA Sample Prep Kit-HI Mammalian (Clontech) following standard instructions. Sequencing was performed on a NextSeq500 instrument (Illumina) with v2 chemistry, resulting in average of $38 \mathrm{M}$ reads per library with $1 \times 75 \mathrm{bp}$ single end setup. The resulting raw reads were assessed for quality, adapter content and duplication rates with FastQC (Andrews S. 2010, FastQC: a quality control tool for high throughput sequence data. Available online at: https://www.bioinforma tics.babraham.ac.uk/projects/fastqc). Trimmomatic version 0.33 was employed to trim reads after a quality drop below a mean of Q20 in a window of five nucleotides (Bolger et al., Trimmomatic: a flexible trimmer for Illumina sequence data). Only reads between 30 and 150 nucleotides were cleared for further analyses. Trimmed and filtered reads were aligned versus the Ensemble human genome version hg38 (GRCh38) using STAR 2.5.2b with the parameter "-outFilterMismatchNoverLmax 0.1 " to increase the maximum ratio of mismatches to mapped length to $10 \%$ (Dobin et al., STAR: ultrafast universal RNA-seq aligner). The number of reads aligning to genes was counted with feature Counts 1.4.5-p1 tool from the Subread package [16]. Only reads mapping at least partially inside exons were admitted and aggregated per gene. Reads overlapping multiple genes or aligning to multiple regions were excluded. Differentially expressed genes were identified using DESeq2 version 1.62 [17]. Adjusted $p$ values were determined by Benjamini-Hochberg correction with a $p$ value of 0.05 considered significant. The Ensembl annotation was enriched with UniProt data (release 06.06.2014) based on Ensemble gene identifiers (Activities at the Universal Protein Resource (UniProt)). The heatmap shows the $Z$ score of each individual replicate of each condition. The $Z$ score was calculated across all replicates for each gene from log-normalized expression. All in the heatmap represented genes are listed in the supplemental Table 5.

\section{ATAC sequencing}

Cells were trypsinized and washed with PBS. Washed cells were counted and 50.000 cells were used for ATAC Library preparation using Tn5 Transposase from Nextera DNA Sample Preparation Kit (Illumina). Cell pellet was resuspended in $50 \mu \mathrm{l} \mathrm{PBS}$ and mixed with $25 \mu \mathrm{l}$ TD-Buffer, $2.5 \mu \mathrm{l} \mathrm{Tn} 5$, $0.5 \mu \mathrm{l} 10 \% \mathrm{NP}-40$ and $22 \mu \mathrm{l}$ water. Cell/Tn5 mixture was incubated at $37^{\circ} \mathrm{C}$ for 30 min with occasional snap mixing. Transposase treatment was followed by $30 \mathrm{~min}$ incubation at $50{ }^{\circ} \mathrm{C}$ together with $500 \mathrm{mM}$ EDTA pH8.0 for optimal recovery of digested DNA fragments. For neutralization of EDTA $100 \mu \mathrm{l}$ of $50 \mathrm{mM} \mathrm{MgCl} 2$ was added followed by purification of the DNA fragments by MinElute PCR Purification Kit (Qiagen). Amplification of Library together with Indexing was performed as described elsewhere [3]. Sequencing, mapping, and read filtering: libraries were mixed in equimolar ratios and sequenced on NextSeq500 platform using V2 chemistry with paired-end mode following assessment for quality using FastQC (Andrews S. 2010, FastQC: a quality control tool for high throughput sequence data. Available online at: https://www.bioinformatics.babraham.ac.uk/proje cts/fastqc). Trimmomatic version 0.33 was employed to trim reads after a quality drop below a mean of Q20 in a window of five nucleotides [2]. Only reads above 30 nucleotides were cleared for further analyses. Reads were mapped versus the hg19 version of the human genome with STAR 2.4.2a [7] using only unique alignments to exclude reads with unclear placing. The reads were further deduplicated using Picard 1.136 (Picard: A set of tools (in Java) for working with next generation sequencing data in the BAM format; https://broad institute.github.io/picard/) to avoid PCR artifacts leading to multiple copies of the same original fragment. Peak calling, filtering, and annotation: For identification of peaks the MUSIC peakcaller (version from December 2015) [9] was employed in punctate mode to accommodate for the 


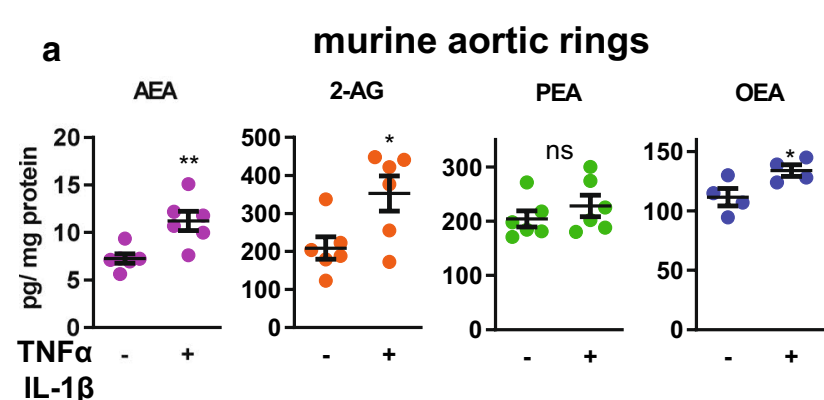

b
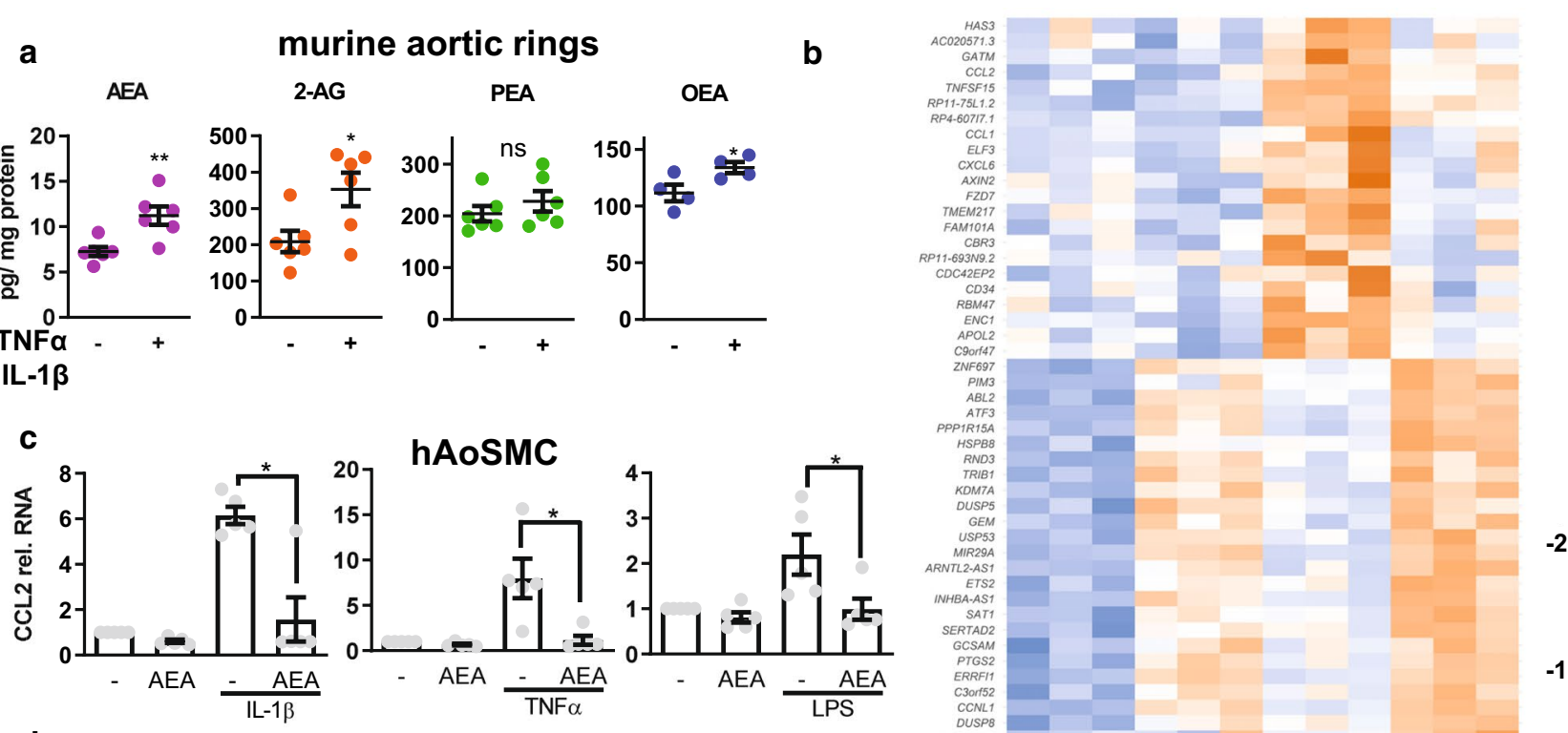

d

mAoSMC

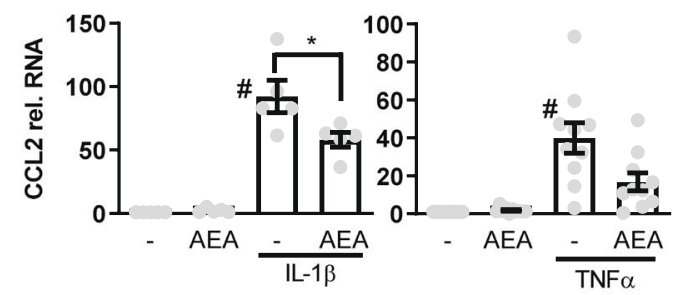

e

murine aortic rings
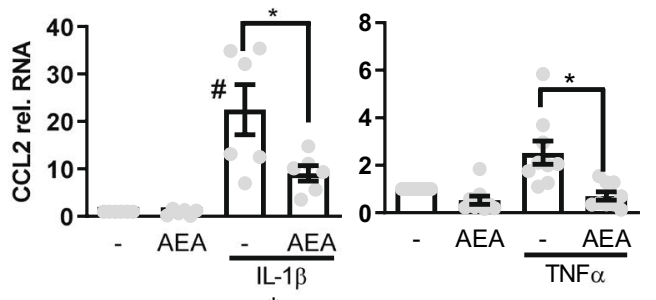

f
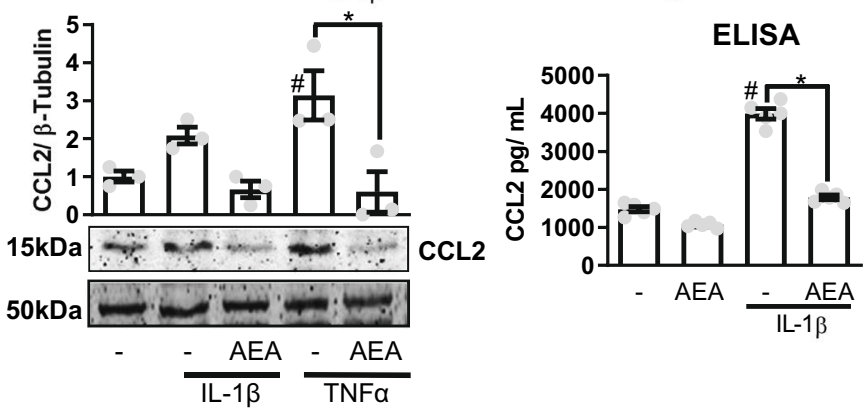

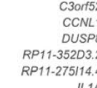

$-1$

1

2

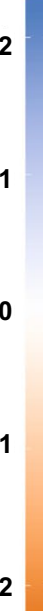

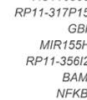

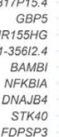

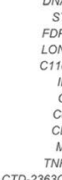

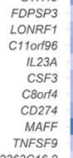

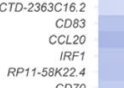

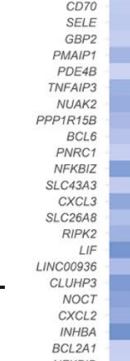

NFKBID

CTL

AEA

IL-1 $\beta \quad$ IL-1 $\beta$ AEA

range of peak widths typically expected for ATAC-seq. Unification of peaks: to compare peaks in different samples, the resulting lists of significant peaks were overlapped and unified to represent identical regions. After conversion of
BAM files to BigWig format with deepTools bamCoverage [28], the counts per unified peak per sample were computed with BigWigAverageOverBed (UCSC Genome Browser Utilities, https://hgdownload.cse.ucsc.edu/downloads.html). 
४Fig. 1 Anandamide (AEA) is released by vascular cells and attenuates induction of a subset of IL-1 $\beta$ responsive genes in HAoSMC. a Cellular concentration of the indicated endocannabinoids as determined by LC-MS/MS from murine aortic rings treated with TNF $\alpha$ and IL-1 $\beta 10 \mathrm{ng} / \mathrm{mL}$ each, $4 \mathrm{~h}$ or with control (PBS with $0.1 \%$ BSA). $N=4-6$, unpaired $t$ test. b Heatmap showing $Z$ score of restricted to IL-1 $\beta$-induced genes with expression significantly altered by $100 \mathrm{nmol} / \mathrm{L}, 150 \mathrm{~min}$ AEA and subsequently $10 \mathrm{ng} / \mathrm{mL} \mathrm{IL}-1 \beta$ for $90 \mathrm{~min}$ in HAoSMC. $N=3$ RT-qPCR of CCL2 performed from human aortic (c) and mouse aortic (d) smooth muscle cells and murine aortic rings (e) in response to the stimuli indicated. Cells treated with $100 \mathrm{nmol} / \mathrm{L} \mathrm{AEA}$ for $150 \mathrm{~min}$ (for tissue: overnight) and subsequently stimulation with $10 \mathrm{ng} / \mathrm{mL}$ cytokine for $90 \mathrm{~min}$. $N=5-10$ f Protein levels of CCL2 as determined by Western blot analysis after treatment with $100 \mathrm{nmol} / \mathrm{L}$ AEA for at least $1 \mathrm{~h}$ and subsequently $10 \mathrm{ng} / \mathrm{mL} \mathrm{IL}-1 \beta$ for $3 \mathrm{~h}$ (normalized to $\beta$-tubulin, $N=3$ ) or ELISA $N=5$. Ordinary one-way ANOVA with Tukey's multiple comparison post-hoc test. If not normally distributed nonparametric ANOVA with Kruskal-Wallis test and Dunn's multiple comparison post-hoc test

Raw counts for unified peaks were submitted to DESeq2 for normalization [1]. Spearman correlations were produced to identify the degree of reproducibility between samples using R. Normalization of samples for IGV: to permit a normalized display of samples in IGV, the raw BAM files were normalized for sequencing depth (number of mapped deduplicated reads per sample) and noise level (number of reads inside peaks versus number of reads not inside peaks). Two factors were computed and applied to the original BAM files using bedtools genomecov resulting in normalized BigWig files for IGV.

\section{Statistics}

Unless otherwise indicated, data are given as means \pm standard error of mean (SEM). Calculations were performed with Prism 8.0 or BiAS.10.12. For multiple group comparisons, ANOVA followed by Tukey's or Sidak's multiple comparison was performed. Data without normal distribution were tested with nonparametric ANOVA followed by Kruskal-Wallis test and Dunn's correction. Individual statistics of unpaired samples were performed by unpaired $t$ test, if not normally distributed with Mann-Whitney test. $p$ values of $<0.05$ were considered as significant. Unless otherwise indicated, $N$ indicates the number of individual experiments.

\section{Source of founding}

This work was supported by grants from the DFG, SFB1039 (TP A01 (RPB), A02 (DS), A06 (IF), B07 (DMzH) and Z01 (GG)), by the Cardio-Pulmonary Institute-CPI and by the Goethe University Frankfurt, Germany.

\section{Data availability}

We have uploaded the RNA-Seq data into the public NCBI GEO database (https://www.ncbi.nlm.nih.gov/geo/query/ acc.cgi .acc $=$ GSE131732).

\section{Results}

\section{AEA attenuates a subset of IL-1 $\beta$ responsive genes in HAOSMC}

To infer an autocrine or paracrine function, the vascular production of endocannabinoids should be dynamic. To test this, murine aortic rings were stimulated with $\mathrm{TNF} \alpha$ and IL- $1 \beta$ or solvent for $4 \mathrm{~h}$ and endocannabinoid production was measured by LC-MS/MS (Fig. 1a). Stimulation with the combination of TNF $\alpha$ and IL-1 $\beta$ cytokines increased the production of AEA and 2-AG as well as OEA, but not PEA in murine aortic tissue, with AEA levels increasing the most significant (Fig. 1a).

Therefore, all subsequent studies focused on this highly important endocannabinoid. To determine whether AEA is relevant for cellular gene expression, human aortic smooth muscle cells (HAoSMC) were pre-stimulated with the endocannabinoid for $150 \mathrm{~min}$ followed by cytokine stimulation for $90 \mathrm{~min}$ and subsequent gene expression analysis by RNA-Seq. This protocol was chosen because $90 \mathrm{~min}$ cytokine stimulation was unable to significantly increase endocannabinoid production (data not shown). Interestingly, AEA had a profound effect on cytokine-mediated gene induction: IL1 $\beta$ induced 409 genes in HAoSMC and AEA potentiated the induction of 92 of them. The induction of 21 IL1 $\beta$-responsive genes was suppressed by AEA (Fig. 1b, top regulated genes; Table 1), among these genes were several important pro-inflammatory cytokines like CXCL6, CCL1 and CCL2 [33]. This suggests that AEA impacts inflammatory signaling and chemokine induction in particular, which is reflected by the gene ontology (GO) analysis (Tables 2, 3).

We chose to investigate this further given the importance of CCL2 release from VSMC in aneurysm and arteriosclerosis development [13]. AEA not only blocked the IL1 $\beta$ mediated CCL2 induction but also TNF $\alpha$ - and LPS-mediated induction, as determined by qRT-PCR (Fig. 1c). Moreover, CCL2 induction was suppressed in both the murine aortic smooth muscle cells (Fig. 1d) and in the intact murine aorta tested by ex vivo experiments (Fig. 1e). Furthermore, AEA prevented cytokine-mediated CCL2 protein induction and secretion, as determined by Western blot and ELISA respectively (Fig. 1f). 
Table 1 List of AEA affected genes

\begin{tabular}{llll}
\hline ENSEMBLE & Gene & Log2 fold change & $p$ value \\
\hline ENSG00000108702 & CCL1 & -2.979705102 & 0.011806624 \\
ENSG00000235505 & CASP17P & -2.846548032 & 0.005539894 \\
ENSG00000163435 & ELF3 & -2.292228757 & 0.037224264 \\
ENSG00000103044 & HAS3 & -1.640698004 & 0.004274255 \\
ENSG00000174059 & CD34 & -1.604414104 & 0.0275882 \\
ENSG00000168646 & AXIN2 & -1.567410529 & 0.012764475 \\
ENSG00000172738 & TMEM217 & -1.376487678 & 0.002107083 \\
ENSG00000155760 & FZD7 & -1.28979261 & 1.18 E-06 \\
ENSG00000163694 & RBM47 & -1.271884075 & 0.046690067 \\
ENSG00000171766 & GATM & -1.270319882 & 0.011426252 \\
ENSG00000124875 & CXCL6 & -1.263261284 & 0.044658625 \\
ENSG00000181634 & TNFSF15 & -1.262629814 & 0.006991752 \\
ENSG00000255521 & AL356215.1 & -1.257583402 & 0.010741181 \\
ENSG00000108691 & CCL2 & -1.247502441 & 0.010281235 \\
ENSG00000229056 & HECW2-AS1 & -1.120083132 & 0.041257119 \\
ENSG00000178882 & RFLNA & -1.038896509 & 0.046389575 \\
ENSG00000213443 & AC007068.1 & -0.952963163 & 0.026106235 \\
ENSG00000149798 & CDC42EP2 & -0.923597937 & 0.045922029 \\
ENSG00000128335 & APOL2 & -0.865889174 & 0.00824209 \\
ENSG00000171617 & ENC1 & -0.86382712 & 0.002088145 \\
ENSG00000159231 & CBR3 & -0.756175822 & 0.039504656 \\
\hline & & &
\end{tabular}

Table 2 Go ontology analysis of AEA affected genes in context to linked diseases

\begin{tabular}{lll}
\hline Index & Disease & $p$ value \\
\hline 1 & Inflammation & 0.00006722 \\
2 & Diffuse cutaneous leishmaniasis & 0.0001415 \\
3 & Lung injury & 0.0001972 \\
4 & Leukemia, mast-cell & 0.0002621 \\
5 & Chronic lung injury & 0.0003361 \\
6 & Angiofibroma & 0.0003904 \\
7 & Thyroid diseases & 0.0003986 \\
8 & Bright disease & 0.0004487 \\
9 & Pulmonary hypertension & 0.000498 \\
10 & Nephritis, interstitial & 0.000511 \\
\hline
\end{tabular}

\section{AEA blocks the IL-1 $\beta$-induced leukocyte attraction}

Since AEA only affected a subset of cytokine-induced genes, we wondered whether the effect was functionally important. Therefore, a Boyden chamber assay was performed to study leukocyte-migration towards smooth muscle cellconditioned medium. Medium from IL- $1 \beta$-conditioned SMC elicited a stronger leukocyte attraction than control medium, and this effect was lost after pre-stimulation of the SMC with AEA (Fig. 2a). Importantly, a CCL2-blocking antibody added to the conditioned medium obtained from SMC stimulated with IL-1 $\beta$ prevented the pro-migratory effect but did not alter that of conditioned medium obtained from SMC pretreated with AEA prior to IL- $1 \beta$ stimulation
Table 3 Go ontology analysis of AEA affected genes on their biological processes

\begin{tabular}{lll}
\hline Index & Biological process & $p$ value \\
\hline 1 & Chemokine-mediated signaling pathway (GO:0070098) & 0.00001674 \\
2 & Neutrophil chemotaxis (GO:0030593) & 0.00002011 \\
3 & Mmulticellular organism development (GO:0007275) & 0.00004792 \\
4 & Chemotaxis (GO:0006935) & 0.00006417 \\
5 & Monocyte chemotaxis (GO:0002548) & 0.0007215 \\
6 & Cellular response to interferon-gamma (GO:0071346) & 0.001012 \\
7 & Inflammatory response (GO:0006954) & 0.001302 \\
8 & Cellular response to interleukin-1 (GO:0071347) & 0.001401 \\
9 & Cellular response to lipopolysaccharide (GO:0071222) & 0.001508 \\
10 & Signal transduction (GO:0007165) & 0.001672 \\
\hline
\end{tabular}




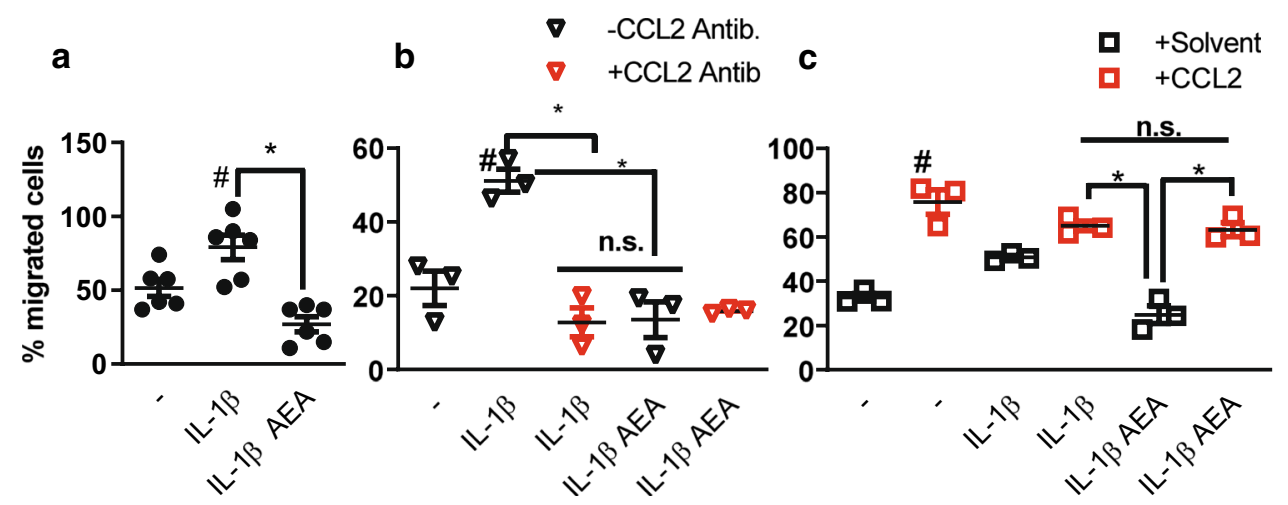

Fig. 2 CCL2 is a functional important target of the inhibitory effect of AEA. a-c Boyden chamber assay with THP1 leukocytic cells and conditioned medium of HAoSMCs treated with or without $100 \mathrm{nmol} / \mathrm{L}$ AEA $(2 \mathrm{~h})$ and $10 \mathrm{ng} / \mathrm{mL} \mathrm{IL-1 \beta}(3 \mathrm{~h})$ as indicated

(Fig. 2b). Finally, addition of recombinant CCL2 to conditioned medium obtained from SMC pretreated with AEA prior to IL-1 $\beta$ stimulation elicited a similar pro-migratory effect as with conditioned medium from IL- $1 \beta$ stimulated SMC. Collectively, these data demonstrate that the effect of AEA is highly important biologically and is mediated by CCL2.

\section{AEA-mediated anti-inflammatory actions are not caused by altered NF-KB/AP-1 signaling}

It is well known that cytokines like TNF $\alpha$ and IL- $1 \beta$ induce inflammatory gene expression through activation of NF- $\mathrm{KB}$ or AP-1. Moreover, it has been shown that NF- $\mathrm{\kappa B}$ and AP-1 are both crucial for CCL2 gene expression [35]. Gene ontology analysis of the 21 AEA-sensitive and IL- $1 \beta$-induced genes recovered a signature containing the RELA subunit of NF- $\mathrm{kB}$ as well as FOS of the AP-1 transcription factors (data not shown). We therefore investigated whether AEA impacts NF- $\mathrm{kB}$ or AP-1 signaling. Compatible with a cannabinoid receptor-dependent activation of $\mathrm{G}_{\mathrm{i}}$-proteins, AEA acutely increased the phosphorylation of p38-MAP Kinase and ERK1/2 (Fig. 3a) but had no effect on the cytokineinduced translocation of the NF- $\mathrm{KB}$ subunit p65 (RELA) into the nucleus (Fig. 3b) or on the formation of the NFKB p50/p65 complex in the nucleus as determined by EMSA (Fig. 3c). These data suggest that AEA increases rather than attenuates the induction of NF- $\mathrm{KB}$ and AP-1-dependent genes. To address this, the RNA-Seq data were screened for genes which are known to be regulated by these transcription factors: CXCL8 [14], TRAF1 [29], TLR2 [13], VCAM-1 [12] and ICAM-1 [4]. As shown in Fig. 3d, most of these genes were induced by AEA itself and AEA did not block the IL-1 $\beta$-mediated induction. Collectively, these data suggest that direct inhibition of AP-1 or NF- $\mathrm{KB}$ is unlikely to (a). Effect of CCL2-neutralizing antibody (b, 1:1000 in condition medium) or CCL2 protein recombinant protein (c, $50 \mathrm{ng} / \mathrm{mL}$ in conditioned medium). $N=3-6,{ }^{\#} p<0.05-v s$. treatment. Ordinary oneway ANOVA with Tukey's multiple comparison post-hoc test

be the mechanism through which AEA mediates its antiinflammatory effect.

\section{AEA treatment inhibits CCL2 expression through HDAC4-dependent gene repression}

Since AEA did not interfere with AP-1 or NF- $\kappa B$ signaling, it might suppress binding of NF- $\kappa B$ to DNA epigenetically. To investigate the accessibility of chromatin at the CCL2 promoter, an assay for Transposase-accessible chromatin using sequencing (ATAC-Seq) was performed. Indeed ATAC-Seq demonstrated that IL- $1 \beta$ induced accessible DNA regions at the CCL2 promoter and that AEA in contrast compacts the chromatin (Fig. 4a).

This was studied further with chromatin immuno-precipitation (ChIP) of the CCL2 promoter. As expected, IL-1 $\beta$ stimulation increased binding of RNA polymerase II and NF-kB p65 to the CCL2 transcriptional start site, in turn activating CCL2 transcription (Fig. 4b). Moreover, histone 3 lysine 4 monomethylation (H3K4me1), which marks promoter inactivation [5] was decreased and $\mathrm{H} 3 \mathrm{~K} 4$ trimethylation (H3K4me3), a mark for transcriptional activation [32] was increased (Fig. 4c). Importantly, all of these IL- $1 \beta$ mediated effects were prevented by AEA pretreatment. Given that nuclear import of NF- $\mathrm{\kappa B}$ was unaffected and that AP-1 target genes were increased with AEA, it would appear that AEA instead activates an independent epigenetic mechanism. We, therefore, investigated the CCL2 H3K27 modification landscape. Interestingly, while IL1 $\beta$ had little impact on the modification of $\mathrm{H} 3 \mathrm{~K} 27$ at the CCL2 TSS, AEA pretreatment increased H3K27me3, a mark for heterochromatin [31] and simultaneously decreased H3K27 acetylation, a mark for promoter activation (Fig. 4d). This suggests that AEA treatment potentially results in the recruitment of a histone 


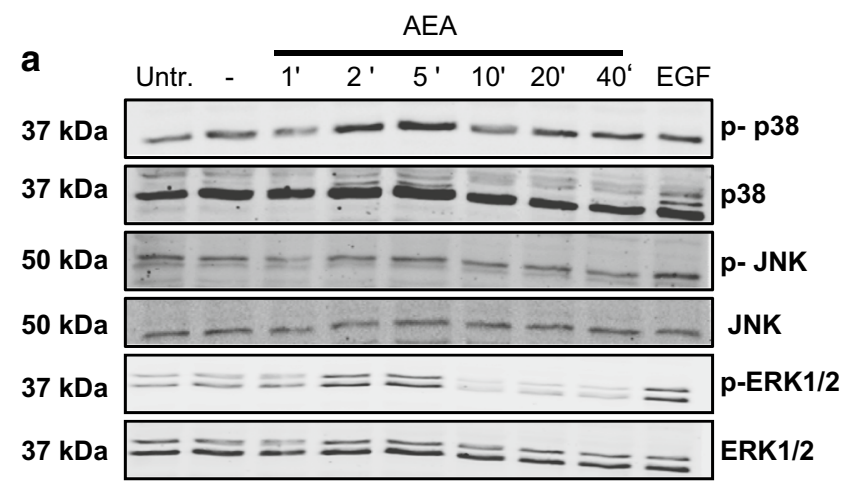

b

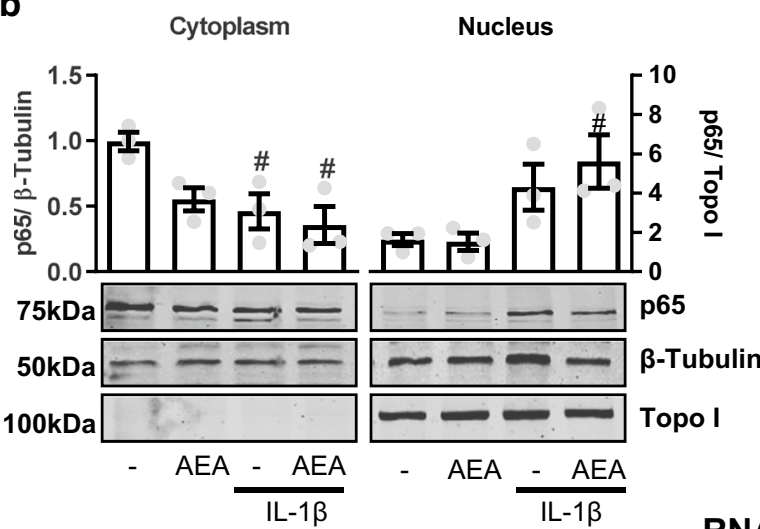

C

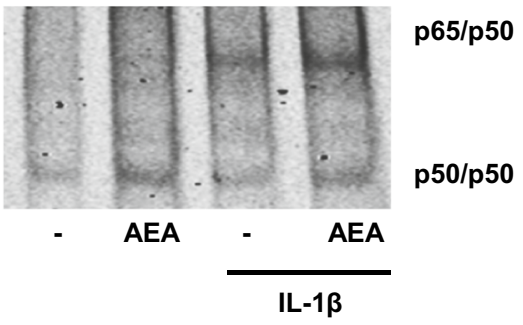

d

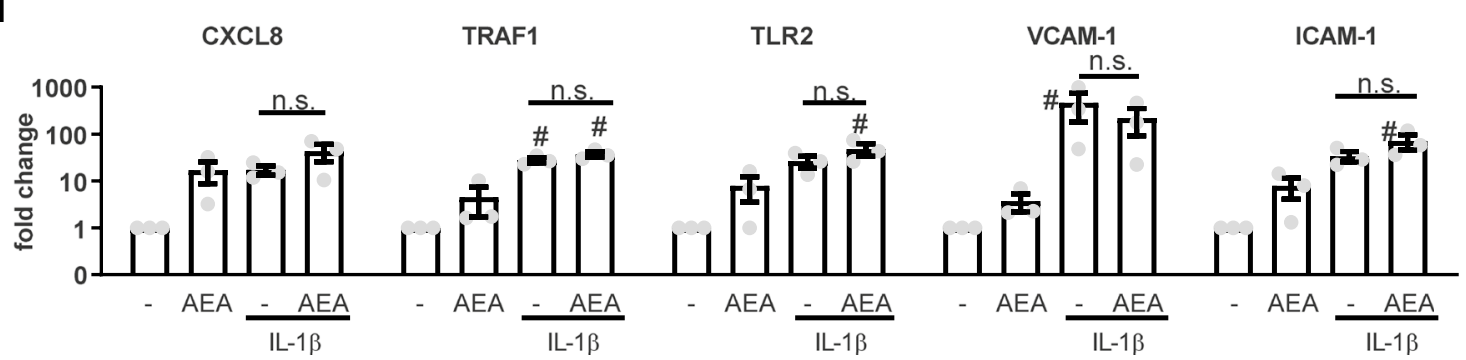

Fig. 3 AEA-mediated anti-inflammatory actions are not mediated by altered NF-kB/AP-1 signaling. a Effect of AEA (100 nmol/L, applied for the minutes indicated) and the positive control EGF (10 ng/ml, $10 \mathrm{~min}$ ) on expression and phosphorylation (indicated by $p$ ) of the MAP kinases shown as detected by Western blot from HAoSMC. Untr. untreated, - solvent control (ethanol 0.05\%). b Western blot analysis of NF- $\kappa \mathrm{B}$ p65 localization in HAoSMC after exposure to IL1 $\beta$ (30 min, $10 \mathrm{ng} / \mathrm{ml})$ and AEA (150 min, $100 \mathrm{nmol} / \mathrm{L}) . N=3$, c Exemplary electrophoretic mobility shift assay (EMSA) for NFKB of

deacetylase (HDAC) to the CCL2 promoter, which leads to epigenetic gene silencing.

To determine whether the observed effect was indeed mediated by HDACs, we first used HDAC inhibitors and measured CCL2 expression. RGFP966, an HDAC class I inhibitor [20], had no effect on AEA-induced CCL2repression, while MC1568, an HDAC class IIa inhibitor [8], completely blocked the AEA-mediated repression of human aortic smooth muscle cells treated with or without AEA (CTL or $100 \mathrm{nmol} / \mathrm{L}, 150 \mathrm{~min}$ ) and subsequently with our without IL-1 $\beta$ (10 ng/mL, $30 \mathrm{~min}$ ). d Expression of known NF-кB/AP-1 target genes from the RNA-Seq data set. Reads are normalized to solvent control to determine fold changes shown on a logarithmic axis. $N=3$. ${ }^{\#} p<0.05$ - vs. treatment. Ordinary one-way ANOVA with Tukey's multiple comparison post-hoc test. If not normally distributed nonparametric ANOVA with Kruskal-Wallis test and Dunn's multiple comparison post-hoc test

CCL2 (Fig. 4e) as well as the AEA-mediated deacetylation of H3K27 (Fig. 4f). The HDAC IIa family consists of four members (HDAC 4, 5, 7 and 9). To determine the specific HDAC responsible for this effect, knockdown experiments with siRNAs against each HDAC class IIa member were performed. Treatment with each siRNA resulted in a more than $70 \%$ reduction in the respective HDAC mRNA expression (Fig. 4g). However, only knockdown 
of HDAC4 blocked the effect of AEA on CCL2 mRNA expression, whereas knockdown of HDAC5 completely blocked the inflammatory response. AEA treatment resulted in the nuclear accumulation of HDAC4 (Fig. 4h) which is in agreement with the fact that class IIa HDACs shuttle between the cytoplasm and nucleus depending on their activation [11]. Together, these data suggest that AEA facilitates nuclear import of HDAC4 and its recruitment to the CCL2 gene locus, where it mediates gene repression.

\section{AEA prevents removal of NCoR1 from the CCL2 promoter}

HDACs are normally recruited to genes by repressors or corepressor complexes [21]. To identify the repressive factor required for AEA-mediated anti-inflammatory actions and HDAC4 recruitment, the RNA-Seq data was reanalyzed for the expression of proteins participating in repressive epigenetic events in HAoSMCs. Several repressors were detected, with NCoR1 and SMRT exhibiting the highest mRNA abundance (Fig. 5a). Knockdown of the five most abundant repressors by siRNA revealed that NCoR 1 is the repressor involved in CCL2 expression (Fig. 5b). Its knockdown increased basal CCL2 expression, completely blocked the inflammatory response and prevented the inhibitory effect of AEA. In contrast, knockdown of the other repressors slightly increased the IL- $1 \beta$ response but had no effect on basal CCL2 expression or the inhibitory effect of AEA. Proximity ligation assays indeed demonstrated a co-localization of NCoR1 and HDAC4 (Fig. 5c). Furthermore, ELISA measurements revealed that NCoR1 knockdown increased basal CCL2 secretion, prevented the effect of IL- $1 \beta$ on CCL2 and blocked the anti-inflammatory effect of AEA (Fig. 5d).

These observations suggest that under basal conditions the CCL2 promoter is suppressed by NCoR 1 and that IL- $1 \beta$ reduces this binding which can be prevented by AEA. To test this, the expression and localization of NCoR1 was studied. Neither AEA nor IL- $1 \beta$ influenced NCoR1 mRNA expression (Fig. 5e). However, IL-1 $\beta$ promoted NCoR1 nuclear export and this effect was prevented by AEA, as observed by Western blot analysis and immuno-fluorescence (Fig. 5f, g). Finally, we wanted to perform NCoR1 immuno-precipitation followed by protein interaction analyses and mass spectrometry. Unfortunately, pulldown of NCoR1 with all available antibodies yielded unsatisfactory results for coimmunoprecipitation experiments. Nevertheless, NCoR1 ChIP revealed the putative site of action on the CCL2 promoter demonstrating that NCoR1 binds to a region $600 \mathrm{bp}$ upstream of the CCL2 transcriptional start site. This interaction was potentiated by AEA treatment (Fig. $5 \mathrm{~h}$ ).

\section{Discussion}

In the present work we demonstrate a novel suppressive effect of the endocannabinoid AEA on a specific subset of inflammatory genes. Pretreatment with AEA elicited a strong anti-inflammatory response through recruitment of the NCoR 1 corepressor complex to the CCL2 locus. Furthermore, AEA promoted the nuclear import of HDAC4 which resides in the nucleus near NCoR1. Thus, in response to AEA, the nuclear corepressor NCoR1 is retained and stabilized at the promoter of CCL2, resulting in suppression of cytokine-induced gene expression by an HDAC4-dependent deacetylation of H3K27.

Despite being somewhat indirect, the interaction between a repressor and HDACs seems to be a fundamental mechanism of gene expression. Although we could demonstrate a close proximity between HDAC4 and $\mathrm{NCoR} 1$ in the nucleus, we failed to demonstrate a direct protein-protein interaction of the two proteins by coimmuno-precipitation or immuno-precipitation followed by mass spectrometry (data not shown). Interestingly, it has recently been suggested in cardiac myocytes that interaction of the two proteins is indeed mediated by a third protein, a member of the MEF2 family [15]. In our own mass spectrometry analysis, we were unable to recover MEF2 as an NCoR1 interaction partner. It therefore seems plausible that a so far unidentified transcription factor mediates this interaction in SMCs. This aspect is likely to be important as AEA only suppressed a subset of IL- $1 \beta$ inducible genes. The ATAC-Seq data suggested that AEA selectively induced chromatin compaction on these genes (like CCL1, CXCL6 and CCL2), whereas chromatin was observed to be open for the genes induced by AEA, like CXCL8 and VCAM-1. It is tempting to speculate that this difference is mediated by binding of NCoR 1 to the DNA. This, however, is difficult to demonstrate, as NCoR 1 does not directly bind DNA and thus does not contain a defined DNA-binding motif. Additionally, numerous transcription factors can participate in DNA binding of a nuclear repressor complex. Despite these considerations, NCoR1 ChIPSeq data has been published (Encode: GSM1003565). Although the quality of this data is suboptimal, due to the points mentioned above, decoration of CCL2, CXCL6 and CCL1 by NCoR1 was observed. Other genes which were unaffected by AEA pretreatment did not contain a clear NCoR1 signature in their promoter region.

In addition to the anti-inflammatory effect acting on CCL2 and some other genes, AEA increased the expression of some other inflammatory genes (see heat map). Some of them contain AP-1 and NFKB binding sites and thus it appears that the function of AEA depends on the gene context. The pro-inflammatory function of AEA is 

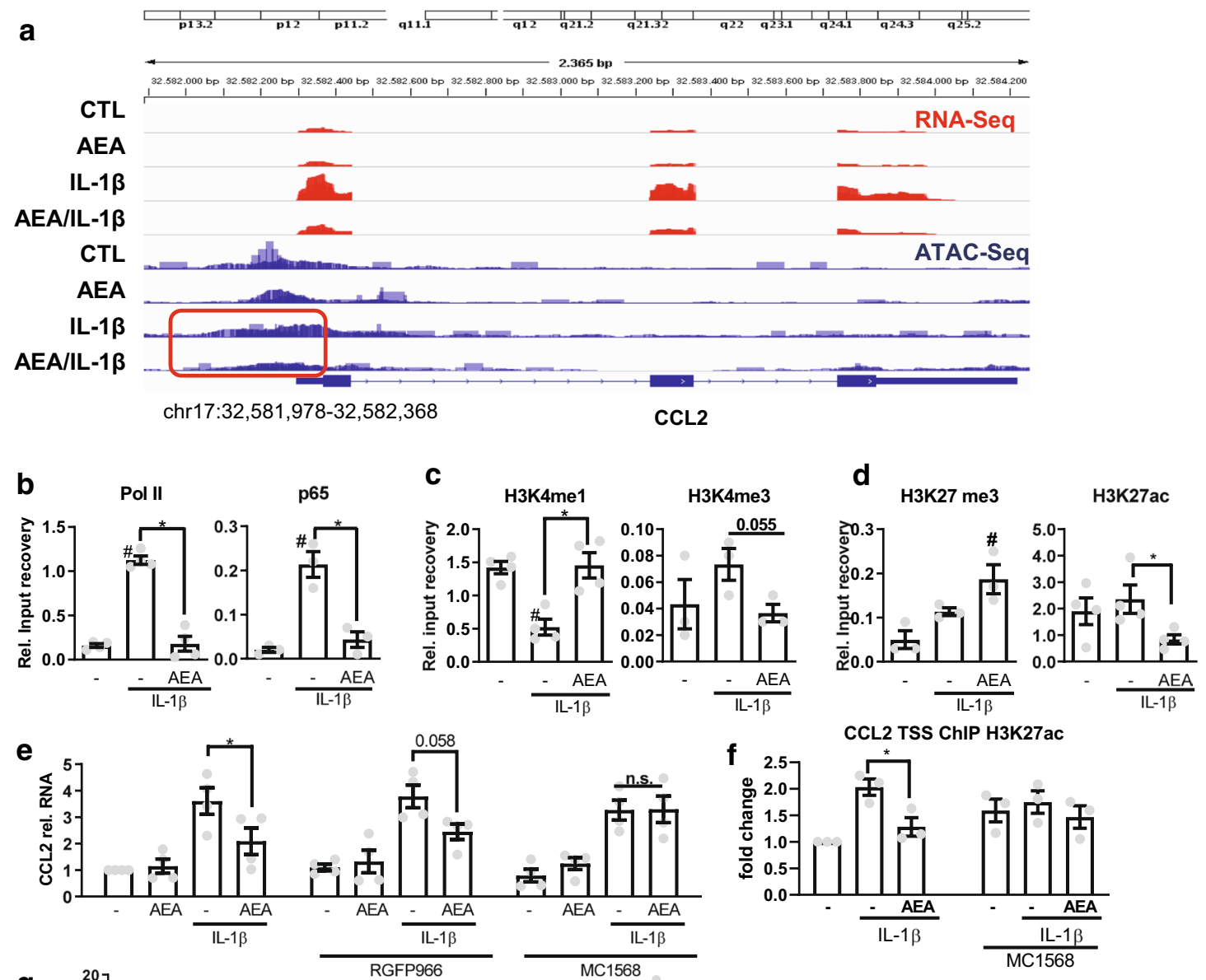

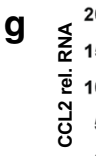

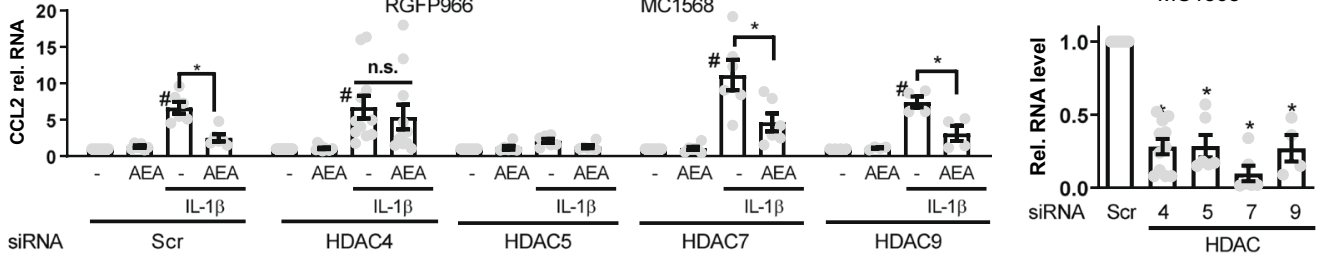

h
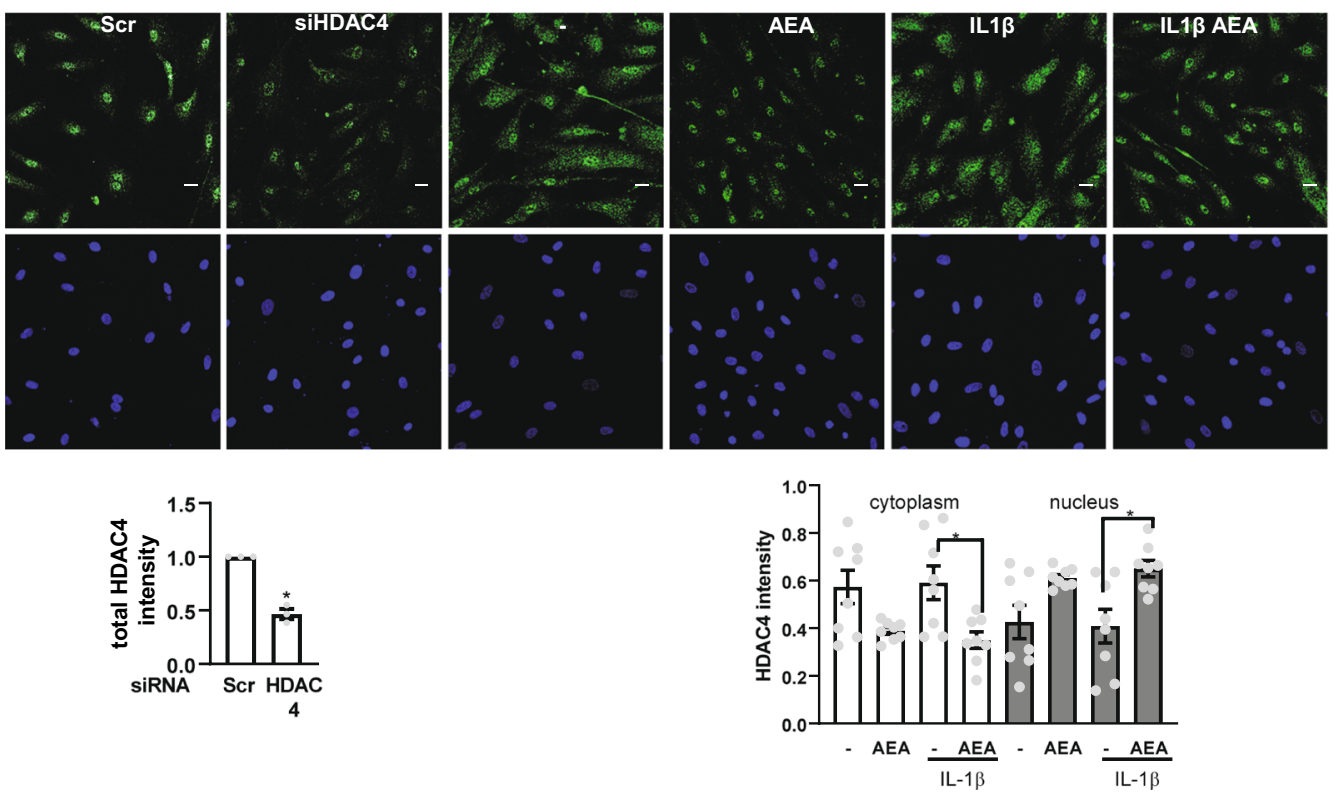
4Fig. 4 AEA-induced CCL2 repression involves HDAC4. a RNASeq and ATAC-Seq channel overly tracing of HAoSMC for the stimuli indicated $(100 \mathrm{nmol} / \mathrm{L}$ AEA, $150 \mathrm{~min}$ und $10 \mathrm{ng} / \mathrm{mL}$, IL-1 $\beta$ $90 \mathrm{~min}$ ). Focusing chromatin structure at the CCL2 Promoter region chr17:32,581,978-32,582,368 in context to IL-1 $\$ \pm$ AEA treatment (red box). $N=3$ Chromatin immunoprecipitation (ChIP) of the proteins indicated followed by qPCR for the CCL2 transcription start site (TSS) in the absence (b-d) or presence (f) of MC1568 $(32 \mu \mathrm{M})$. e CCL2 RT-qPCR of HAoSMC pretreated with the HDAC inhibitor RGFP966 $(2.7 \mu \mathrm{M})$ or MC1568 $(32 \mu \mathrm{M})$ or solvent (DMSO) for $1 \mathrm{~h}$ and afterwards stimulated with AEA (100 nmol/L $150 \mathrm{~min})$ and IL-1 $\beta$ (10 ng/mL $90 \mathrm{~min}$; for ChIP experiments: $120 \mathrm{~min} 100 \mathrm{nmol} / \mathrm{L}$ AEA and $10 \mathrm{ng} / \mathrm{mL}$ IL-1 $\beta 60 \mathrm{~min}$ ). Two-way ANOVA with Tukey's multiple comparison post-hoc test. g CCL2 (left), HDAC (right) RT-qPCR after siRNA knockdown of the HDACs indicated (40 nM, $72 \mathrm{~h}$ ). Normalized to each siRNA untreated (-). $N=4-11$, two-way ANOVA with Tukey's multiple comparison post-hoc test. h Immunofluorescence of HDAC4 in HAoSMC after treatment with AEA $(100 \mathrm{nmol} / \mathrm{L}, 150 \mathrm{~min})$ and IL-1 $\beta(10 \mathrm{ng} / \mathrm{mL}, 60 \mathrm{~min})$ or HDAC4 siRNA. Scale bar $20 \mu \mathrm{m}, N=8$. Immunofluorescence after siRNA knockdown (40 nM, 72 h) $n=3$, unpaired $t$ test. ${ }^{*} p<0.05-$ vs. treatment, $* p<0.05$. Ordinary one-way ANOVA with Tukey's multiple comparison post-hoc test. If not normally distributed nonparametric ANOVA with Kruskal-Wallis test and Dunn's multiple comparison post-hoc test

not mediated by NCoR-1 (data not shown) so that we did not address further this aspect in the present work.

In the present study, we could demonstrate that AEA is released from the murine aorta in response to cytokine stimulation and that exogenously-applied AEA strongly prevents cytokine-mediated CCL2 induction. This mechanism strongly suppresses cytokine-mediated CCL2 induction and thereby prevents leukocyte chemotaxis. It is, however, unclear whether the endogenous production of AEA is sufficient to elicit a similar response. Although AEA is considered a very important endocannabinoid, it is somewhat unstable due to its metabolism by several enzymes. It is believed that AEA is formed from $N$-arachidonoyl-phosphatidylethanolamine (NAPE) by the action of NAPE-PLD. Besides this there is evidence that AEA is also formed by enzymatic activity of other enzymes like $\mathrm{PLA}_{2}, \mathrm{PLC} / \mathrm{PTPN} 22$ and ABHD4/GDE1. NAPE itself is produced by several $N$-acyltransferases [19]. The redundancy in this pathway makes approaches with knockout mice specific for the individual enzyme unfeasible. The same limitation applies for AEA degradation, which can occur through many different pathways involving the P450 monooxygenases and lipoxygenases [19], which are all expressed in the vascular system. We were unable to increase vascular AEA levels using cyclooxygenase inhibition and knockdown cells targeting the degradation pathways to arachidonic acid by FAAH and NAAA (data not shown). Therefore, the present work reports a novel interesting pharmacological pathway but its exploitation will depend on the possibility to generate AEA mimetics.

In conclusion, we have identified a novel potent antiinflammatory epigenetic mechanism for the endogenous endocannabinoid anandamide (AEA). AEA mimetics could be developed as potent tools to limit chemotactic recruitment of monocytic cells into areas of inflammation. 


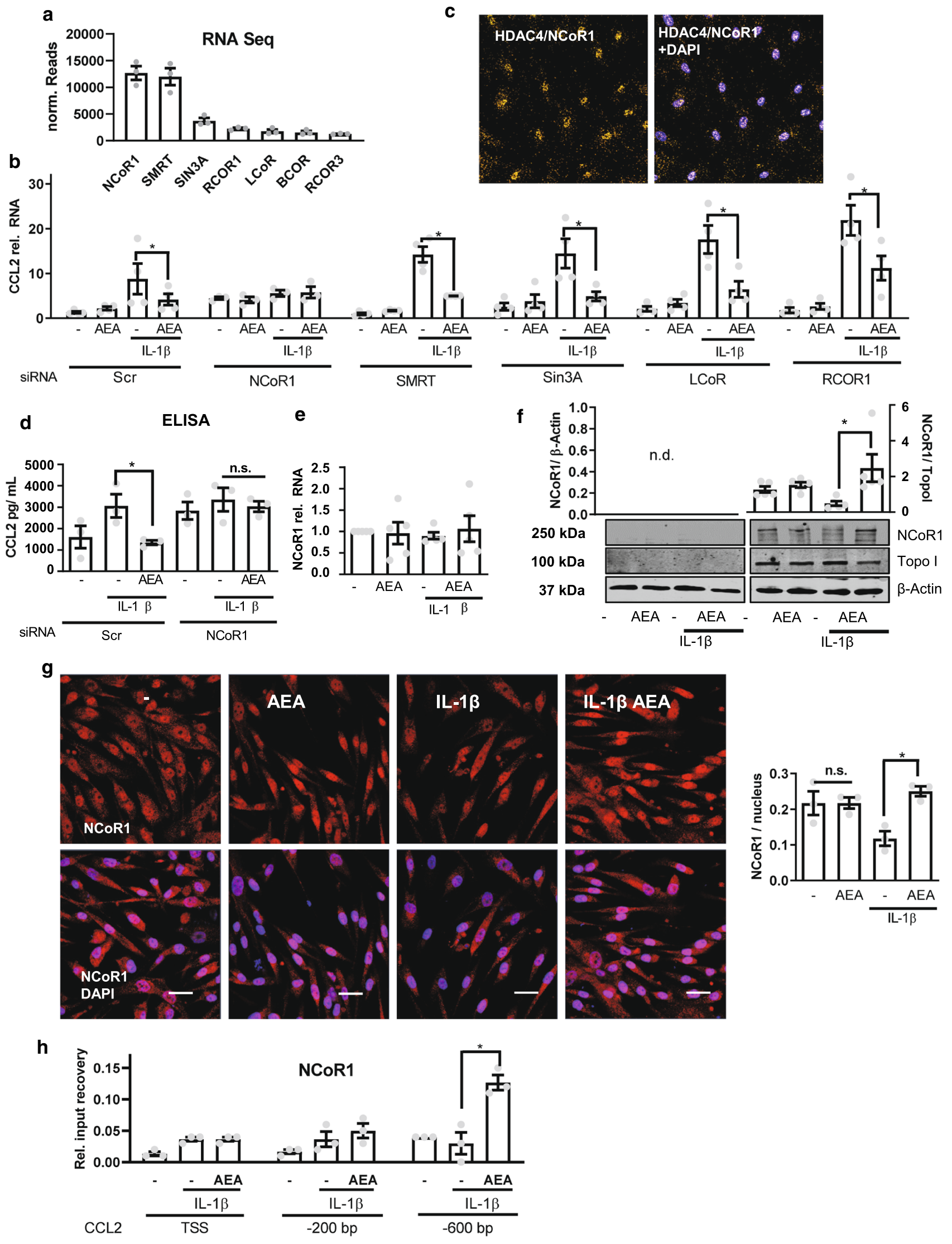


४Fig. 5 NCOR1 mediates AEA-induced CCL2 suppression. a mRNA expression of repressors in HAoSMC from the RNA-Seq dataset normalized by median of ratios. $N=3$. b CCL2 RT-qPCR in HAoSMC after siRNA knockdown of the repressors indicated ( $40 \mathrm{nM}, 72 \mathrm{~h}$ ). Scr denotes the scrambled siRNA control. Normalized to siRNA Scr. Treatment: $100 \mathrm{nmol} / \mathrm{L}$ AEA, $150 \mathrm{~min}$ and IL-1 $\beta 10 \mathrm{ng} / \mathrm{mL}$ 90 min. $N=3-4$, two-way ANOVA with Tukey's multiple comparison post-hoc test. c Proximity ligation assay in HAoSMC for HDAC4 and NCoR1 after treatment $100 \mathrm{nmol} / \mathrm{L}$ AEA, $150 \mathrm{~min}$ and $10 \mathrm{ng} /$ $\mathrm{mL}$ IL- $1 \beta 60 \mathrm{~min}, N=4$. d CCL2 protein levels measured by ELISA with and without siRNA-mediated knockdown of NCoR1 or Scr as control. Media was collected after $4 \mathrm{~h}$ as described before. Two-way ANOVA with Tukey's multiple comparison post-hoc test. e NCoR1 expression as determined by RT-qPCR of HAoSMC. $N=5$. f Western blot analysis of HAoSMC nuclear extracts for NCoR1 after treatment with $100 \mathrm{nmol} / \mathrm{L}$ AEA $150 \mathrm{~min}$ and $60 \mathrm{~min} 10 \mathrm{ng} / \mathrm{mL} \mathrm{IL-1 \beta .} N=5$. g Immunofluorescence of NCoR1 in HAoSMC treated similar to (f). Scale bar $=50 \mu \mathrm{m}, N=4$. h ChIP analysis of NCoR 1 in HAoSMC $n=4$. Treatment $120 \mathrm{~min} 100 \mathrm{nmol} / \mathrm{L}$ AEA and $10 \mathrm{ng} / \mathrm{mL}$ IL- $1 \beta$ 60 min. $* p<0.05$. Ordinary one-way ANOVA with Tukey's multiple comparison post-hoc test. If not normally distributed nonparametric ANOVA with Kruskal-Wallis test and Dunn's multiple comparison post-hoc test

Acknowledgements Open Access funding provided by Projekt DEAL. The authors are grateful for the excellent technical assistance of Susanne Wienstroer, Tanja Lüneburg, Katalin Pálfi and Yannick Schreiber.

\section{Compliance with ethical standards}

Conflict of interest The authors declare that they have no conflict of interest.

Open Access This article is licensed under a Creative Commons Attribution 4.0 International License, which permits use, sharing, adaptation, distribution and reproduction in any medium or format, as long as you give appropriate credit to the original author(s) and the source, provide a link to the Creative Commons licence, and indicate if changes were made. The images or other third party material in this article are included in the article's Creative Commons licence, unless indicated otherwise in a credit line to the material. If material is not included in the article's Creative Commons licence and your intended use is not permitted by statutory regulation or exceeds the permitted use, you will need to obtain permission directly from the copyright holder. To view a copy of this licence, visit http://creativecommons.org/licenses/by/4.0/.

\section{References}

1. Anders S, Huber W (2010) Differential expression analysis for sequence count data. Genome Biol 11:R106. https://doi. org/10.1186/gb-2010-11-10-r106

2. Bolger AM, Lohse M, Usadel B (2014) Trimmomatic: a flexible trimmer for illumina sequence data. Bioinformatics 30:2114 2120. https://doi.org/10.1093/bioinformatics/btu170

3. Buenrostro JD, Giresi PG, Zaba LC, Chang HY, Greenleaf WJ (2013) Transposition of native chromatin for fast and sensitive epigenomic profiling of open chromatin, DNA-binding proteins and nucleosome position. Nat Methods 10:1213-1218. https://doi. org/10.1038/nmeth.2688

4. Bunting K, Rao S, Hardy K, Woltring D, Denyer GS, Wang J, Gerondakis S, Shannon MF (2007) Genome-wide analysis of gene expression in T cells to identify targets of the NF-kappa B transcription factor c-Rel. J Immunol 178:7097-7109. https://doi. org/10.4049/jimmunol.178.11.7097

5. Cheng J, Blum R, Bowman C, Hu D, Shilatifard A, Shen S, Dynlacht BD (2014) A role for H3K4 monomethylation in gene repression and partitioning of chromatin readers. Mol Cell 53:979-992. https://doi.org/10.1016/j.molcel.2014.02.032

6. DevaneWA DIIIFA, Johnson MR, Melvin LS, Howlett AC (1988) Determination and characterization of a cannabinoid receptor in rat brain. Mol Pharmacol 34:605-613

7. Dobin A, Davis CA, Schlesinger F, Drenkow J, Zaleski C, Jha S, Batut P, Chaisson M, Gingeras TR (2013) STAR: ultrafast universal RNA-seq aligner. Bioinformatics 29:15-21. https://doi. org/10.1093/bioinformatics/bts635

8. Fleming CL, Ashton TD, Gaur V, McGee SL, Pfeffer FM (2014) Improved synthesis and structural reassignment of MC1568: a class IIa selective HDAC inhibitor. J Med Chem 57:1132-1135. https://doi.org/10.1021/jm401945k

9. Harmanci A, Rozowsky J, Gerstein M (2014) MUSIC: identification of enriched regions in ChIP-Seq experiments using a mappability-corrected multiscale signal processing framework. Genome Biol 15:474. https://doi.org/10.1186/s13059-014-0474-3

10. Howlett AC, Reggio PH, Childers SR, Hampson RE, Ulloa NM, Deutsch DG (2011) Endocannabinoid tone versus constitutive activity of cannabinoid receptors. Br J Pharmacol 163:1329-1343. https://doi.org/10.1111/j.1476-5381.2011.01364.x

11. Hsu K-C, Liu C-Y, Lin TE, Hsieh J-H, Sung T-Y, Tseng H-J, Yang J-M, Huang W-J (2017) Novel class IIa-selective histone deacetylase inhibitors discovered using an in silico virtual screening approach. Sci Rep 7:3228. https://doi.org/10.1038/s41598-01703417-1

12. Iademarco MF, McQuillan JJ, Rosen GD, Dean DC (1992) Characterization of the promoter for vascular cell adhesion molecule-1 (VCAM-1). J Biol Chem 267:16323-16329

13. Johnson CM, Tapping RI (2007) Microbial products stimulate human Toll-like receptor 2 expression through histone modification surrounding a proximal NF-kappaB-binding site. J Biol Chem 282:31197-31205. https://doi.org/10.1074/jbc.M705151200

14. Kang H-B, Kim Y-E, Kwon H-J, Sok D-E, Lee Y (2007) Enhancement of NF-kappaB expression and activity upon differentiation of human embryonic stem cell line SNUhES3. Stem Cells Dev 16:615-623. https://doi.org/10.1089/scd.2007.0014

15. Li C, Sun X-N, Chen B-Y, Zeng M-R, Du L-J, Liu T, Gu H-H, Liu Y, Li Y-L, Zhou L-J, Zheng X-J, Zhang Y-Y, Zhang W-C, Liu Y, Shi C, Shao S, Shi X-R, Yi Y, Liu X, Wang J, Auwerx J, Wang ZV, Jia F, Li R-G, Duan S-Z (2019) Nuclear receptor corepressor 1 represses cardiac hypertrophy. EMBO Mol Med 11:e9127. https ://doi.org/10.15252/emmm.201809127

16. Liao Y, Smyth GK, Shi W (2014) featureCounts: an efficient general purpose program for assigning sequence reads to genomic features. Bioinformatics 30:923-930. https://doi.org/10.1093/ bioinformatics/btt656

17. Love MI, Huber W, Anders S (2014) Moderated estimation of fold change and dispersion for RNA-seq data with DESeq2. Genome Biol 15:550. https://doi.org/10.1186/s13059-014-0550-8

18. Lu H-C, Mackie K (2016) An introduction to the endogenous cannabinoid system. Biol Psychiatry 79:516-525. https://doi. org/10.1016/j.biopsych.2015.07.028

19. Maccarrone $M$ (2017) Metabolism of the endocannabinoid anandamide: open questions after 25 years. Front Mol Neurosci 10:166. https://doi.org/10.3389/fnmol.2017.00166

20. Malvaez M, McQuown SC, Rogge GA, Astarabadi M, Jacques V, Carreiro S, Rusche JR, Wood MA (2013) HDAC3-selective inhibitor enhances extinction of cocaine-seeking behavior in a persistent manner. Proc Natl Acad Sci USA 110:2647-2652. https ://doi.org/10.1073/pnas.1213364110 
21. Mottis A, Mouchiroud L, Auwerx J (2013) Emerging roles of the corepressors NCoR1 and SMRT in homeostasis. Genes Dev 27:819-835. https://doi.org/10.1101/gad.214023.113

22. Munro S, Thomas KL, Abu-Shaar M (1993) Molecular characterization of a peripheral receptor for cannabinoids. Nature 365:6165. https://doi.org/10.1038/365061a0

23. Navarrete M, Araque A (2008) Endocannabinoids mediate neuron-astrocyte communication. Neuron 57:883-893. https://doi. org/10.1016/j.neuron.2008.01.029

24. Netherland CD, Pickle TG, Bales A, Thewke DP (2010) Cannabinoid receptor type 2 (CB2) deficiency alters atherosclerotic lesion formation in hyperlipidemic Ldlr-null mice. Atherosclerosis 213:102-108. https://doi.org/10.1016/j.atherosclerosis .2010 .07 .060

25. O'Sullivan SE (2007) Cannabinoids go nuclear: evidence for activation of peroxisome proliferator-activated receptors. Br J Pharmacol 152:576-582. https://doi.org/10.1038/sj.bjp.0707423

26. Pacher P, Steffens S (2009) The emerging role of the endocannabinoid system in cardiovascular disease. Semin Immunopathol 31:63-77. https://doi.org/10.1007/s00281-009-0145-8

27. Pacher P, Steffens S, Haskó G, Schindler TH, Kunos G (2018) Cardiovascular effects of marijuana and synthetic cannabinoids: the good, the bad, and the ugly. Nat Rev Cardiol 15:151-166. https ://doi.org/10.1038/nrcardio.2017.130

28. Ramírez F, Dündar F, Diehl S, Grüning BA, Manke T (2014) deepTools: a flexible platform for exploring deep-sequencing data. Nucleic Acids Res 42:W187-W191. https://doi.org/10.1093/nar/ gku365

29. Schwenzer R, Siemienski K, Liptay S, Schubert G, Peters N, Scheurich P, Schmid RM, Wajant H (1999) The human tumor necrosis factor (TNF) receptor-associated factor 1 gene (TRAF1) is up-regulated by cytokines of the TNF ligand family and modulates TNF-induced activation of NF-kappaB and c-Jun N-terminal kinase. J Biol Chem 274:19368-19374. https://doi. org/10.1074/jbc.274.27.19368

30. Steffens S, Veillard NR, Arnaud C, Pelli G, Burger F, Staub C, Karsak M, Zimmer A, Frossard J-L, Mach F (2005) Low dose oral cannabinoid therapy reduces progression of atherosclerosis in mice. Nature 434:782-786. https://doi.org/10.1038/nature0338 9

31. Thakore PI, Black JB, Hilton IB, Gersbach CA (2016) Editing the epigenome: technologies for programmable transcription and epigenetic modulation. Nat Methods 13:127-137. https://doi. org/10.1038/nmeth.3733

32. Tie F, Banerjee R, Stratton CA, Prasad-Sinha J, Stepanik V, Zlobin A, Diaz MO, Scacheri PC, Harte PJ (2009) CBP-mediated acetylation of histone $\mathrm{H} 3$ lysine 27 antagonizes Drosophila polycomb silencing. Development 136:3131-3141. https://doi.org/10.1242/ dev.037127

33. van Damme J, Wuyts A, Froyen G, van Coillie E, Struyf S, Billiau A, Proost P, Wang JM, Opdenakker G (1997) Granulocyte chemotactic protein-2 and related CXC chemokines: from gene regulation to receptor usage. J Leukoc Biol 62:563-569

34. Varga K, Lake KD, Huangfu D, Guyenet PG, Kunos G (1996) Mechanism of the hypotensive action of anandamide in anesthetized rats. Hypertension 28:682-686. https://doi.org/10.1161/01. HYP.28.4.682

35. Wolter S, Doerrie A, Weber A, Schneider H, Hoffmann E, von der Ohe J, Bakiri L, Wagner EF, Resch K, Kracht M (2008) c-Jun controls histone modifications, NF-kappaB recruitment, and RNA polymerase II function to activate the $\mathrm{ccl} 2$ gene. Mol Cell Biol 28:4407-4423. https://doi.org/10.1128/MCB.00535-07 